\title{
EFFECT OF BREED ON MICROSTRUCTURE AND TENDERNESS OF PORCINE SEMIMEMBRANOSUS MUSCLE*
}

\author{
Dorota Wojtysiak \\ Department of Reproduction and Animal Anatomy, Agricultural University of Krakow, \\ Al. Mickiewicza 24/28, 30-059 Kraków, Poland \\ Corresponding author: wojtysiakd@wp.pl
}

\begin{abstract}
The objective of the study was to analyse the effect of breed on characteristics of muscle fibres, parameters of intramuscular connective tissue, collagen content, intramuscular fat (IMF) content and tenderness of semimembranosus muscle in pigs. The study was conducted with 16 Zlotnicka Spotted (ZS) and 23 Polish Landrace (PL) fattening pigs slaughtered at 210 days of age. The current study showed that compared to the PL breed, the semimembranosus muscle from the local breed of ZS pigs was characterized by a greater percentage of type I fibres, a smaller percentage of type IIB fibres and a smaller diameter of all the three muscle fibre types analysed, while the muscles of PL pigs had significantly thinner endomysium and perimysium and a more compact arrangement of collagen fibres, with a significantly lower percentage of collagen in the muscle and a lower content of total and soluble collagen compared to the muscles of ZS pigs. Furthermore, meat from ZS pigs was more tender (lower shear force value) and had a significantly higher IMF content compared to meat from PL pigs.
\end{abstract}

Key words: breed, microstructure, tenderness, $m$. semimembranosus, pigs

Tenderness is generally considered by consumers to be the most important eating quality characteristic of meat. Meat tenderness is influenced by several factors such as age, sex, diet, and preslaughter handling. However, the main determinant of meat tenderness is muscle structure (Ruusunen and Puolanne, 1997; Gil et al., 2008). Tenderness is influenced most by muscle protein components (intramuscular connective tissue, muscle fibres, adipose tissue) in addition to parameters such as $\mathrm{pH}$ of meat, sarcomere length, and activity of the calpain enzyme system. Although intramuscular connective tissue forms only a small portion of the muscle mass, it has a disproportionately large effect on meat tenderness (Nishimura et al., 1996; Fang et al., 1999; Lepetit, 2008). For many years, pigs were selected for improved fattening and slaughter traits, in particular for increased meatiness. This has increased the inci-

\footnotetext{
*This study was supported by the grant No. N311 086034 and DS-3253.
} 
dence of meat quality defects in the most desirable part of the pig carcass, namely the longissimus dorsi muscle, and in one of most representative pork cuts, namely the ham, of which m. semimembranosus is analysed the most often (Wegner et al., 1993). Much hope is placed on the investigations of muscle microstructure to explain the increasing meat content and the deteriorating quality of pig meat. Animal breed is one of the major factors affecting the physicochemical parameters of meat. Accordingly, one way to improve pork quality is to increase the interest in native pig breeds, including the Złotnicka Spotted which represents a valuable genetic resource and is characterized by high resistance to infectious agents, low feed requirements, and very good quality of meat (Florowski et al., 2006; Bogucka and Kapelański, 2005). Therefore, the aim of the current study was to investigate the effect of breed on muscle fibre characteristics, parameters of intramuscular connective tissue, collagen content, intramuscular fat (IMF) content and tenderness of $m$. semimembranosus in pigs.

\section{Material and methods}

The study was performed with 18 Złotnicka Spotted (ZS) and 23 Polish Landrace (PL) pigs with an average body weight of $101.3 \pm 1.06 \mathrm{~kg}$ and $120.9 \pm 2.18 \mathrm{~kg}$, and average $m$. semimembranosus weight of $1.44 \pm 0.06$ and $1.72 \pm 0.14$, respectively. A complete feed for fattening pigs (T-221 Grower Standard) was offered ad libitum from automatic feeders (Normy Żywienia Świń, 1993). All animals were reared under the same environmental and production regime. Pigs were slaughtered at 210 days of age in a commercial slaughterhouse. Feed was withdrawn $12 \mathrm{~h}$ before slaughter but water was freely available in lairage. The pigs were stunned with $\mathrm{CO}_{2}$ and processed according to the normal slaughterhouse procedures. For microstructural examination muscle samples were taken 45 min postmortem from the right carcass side deep within the $m$. semimembranosus and frozen in liquid nitrogen $\left(-196^{\circ} \mathrm{C}\right)$ and stored at $-80^{\circ} \mathrm{C}$ until histochemical analysis. Serial transverse sections of $10 \mu \mathrm{m}$ were cut at $-20^{\circ} \mathrm{C}$ in a cryostat (Slee MEV, Germany). Muscle fibre type (I, IIA and IIB) was distinguished using a modified combined method of NADH-tetrazolium reductase activity and immunohistochemical determination of the slow myosin heavy chain on the same section with monoclonal antibodies against the skeletal slow myosin heavy chain was performed for $1 \mathrm{~h}$ at room temperature (clone WB-MHCs Leica, Germany, dilution 1:80) (Wojtysiak and Kaczor, 2011). Moreover, frozen sections were immunohistochemically stained with rabbit polyclonal antibodies against type I-V collagens (ab27117, Abcam, 1:1000 diluted) to determine connective tissue (collagen fibres). All immunohistochemical reactions were visualized by NovoLink ${ }^{\mathrm{TM}}$ Polymer Detection System (Leica, Germany), according to the manufacturer's instruction. A minimum of 10 images (each $4 \mathrm{~mm}^{2}$ ) were counted in each section using a Zeiss Axio Vision A.2 light microscope. Analysis was made of the thickness of endomysium and perimysium (secondary), and percentage of the collagen area (total perimysium+endomysium) in muscle structure was determined using an image analysis system (Axio Vision Rel. 4.8.3, Zeiss, Germany). Additionally, a minimum 
of 300 fibres were examined from each cross-section to determine muscle fibre percentage and diameter.

Moreover, to examine the collagen fibre architecture in the intramuscular connective tissue of $m$. semimembranosus, muscle samples collected $45 \mathrm{~min}$ postmortem were fixed in $2.5 \%$ glutaraldehyde solution in phosphate buffer $0.1 \mathrm{M}(\mathrm{pH} \mathrm{7.2)}$ for two days at $4^{\circ} \mathrm{C}$. After washing the muscle fragments three times in $0.1 \mathrm{M}$ PBS solution for 2 hours, muscle fibres were macerated in $2 \mathrm{~N} \mathrm{NaOH}$ solution for $6-8$ days at room temperature according to the procedure described by Iwamoto et al. (2001). The architecture of collagen fibres of $m$. semimembranosus was examined under a scanning electron microscope JSM-5410 (a gift from the Foundation for Polish Science SUBIN 94) at an accelerating voltage of $15 \mathrm{kV}$ at the Scanning Microscopy Laboratory of the Faculty of Biology and Earth Sciences of the Jagiellonian University in Kraków.

The intramuscular fat content (IMF) of the $m$. semimembranosus was determined in duplicate according to the procedure recommended by AOAC No. 991.36 (AOAC, 2000), while hydroxyproline content was determined in accordance with the Polish Standard PN-ISO 3496/2000. A factor of 7.25 was used to convert hydroxyproline to total collagen. Insoluble collagen content was determined according to Liu et al. (1996) with modifications. Approximately $5 \mathrm{~g}$ of meat sample was homogenized with $24 \mathrm{~cm}^{3}$ of distilled water. The homogenate was heated in a water bath at $77^{\circ} \mathrm{C}$ for $70 \mathrm{~min}$ and centrifuged in a Janetzky K-24 centrifuge at $10000 \mathrm{rpm}$ for $20 \mathrm{~min}$. The supernatant was decanted and the pellet was washed with distilled water and centrifuged as above. The pellet was hydrolyzed with $30 \mathrm{~cm} 3$ of $3 \mathrm{M} \mathrm{H}_{2} \mathrm{SO}_{4}$ at $105^{\circ} \mathrm{C}$ for $16 \mathrm{~h}$. Further procedures were the same as for the determination of total collagen. The amount of soluble collagen was calculated from the difference between total and insoluble collagen, and expressed as percentage of total collagen. Meat samples for Warner-Bratzler shear force (WB) were taken after $24 \mathrm{~h}$ of cooling at $4^{\circ} \mathrm{C}$. Next, the chops were roasted at $180^{\circ} \mathrm{C}$ to reach an internal temperature of $78^{\circ} \mathrm{C}$ and then cooled to room temperature and weighed for thermal loss determination. Then, five $14 \mathrm{~mm}$ diameter cores were taken from each chop parallel to the muscle fibre orientation. Shear force was measured using a Texture Analyser TA-XT2 (Stable-Micro Systems, UK) with a Warner-Bratzler unit and a triangular blade.

Differences among the breed groups of pigs (main effect) were analysed using analysis of variance (General Linear Models procedure). Slaughter weight was included in the model as a covariate for IMF level. A probability of $\mathrm{P}<0.05$ was considered statistically significant. The data were expressed as least squares means (LSM) \pm standard deviation (SD).

\section{Results}

Table 1 gives measurement results of muscle fibre and connective tissue parameters, the percentage and content of total and soluble collagen, IMF content, and shear force value of porcine $m$. semimembranosus depending on the breed. The results of microstructural analysis are presented in Figure $1 \mathrm{~A}-\mathrm{H}$. 

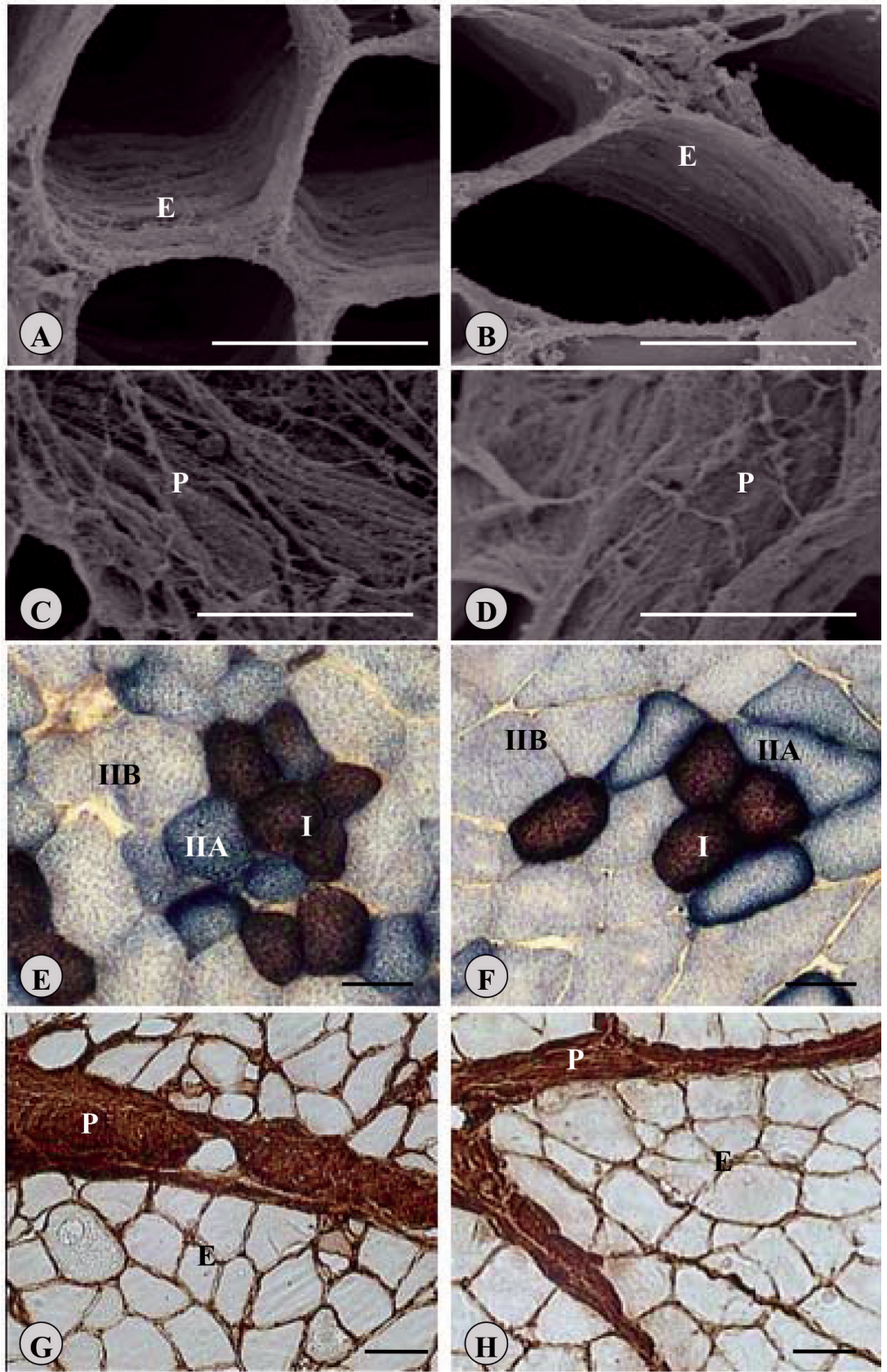

Figure 1. Exemplary cross-sections of $m$. semimembranosus of Złotnicka Spotted (A, C, E, G) and Polish Landrace (B, D, F, H) pigs: SEM electronograms (A, B, C, D); E - endomysium;

$\mathrm{P}$ - perimysium (secondary); NADH-TH and immunohistochemical MHC slow staining (E, F); I - red fibres; IIA - intermediate fibres; IIB - white fibres; immunohistochemical detection of type I-V collagen $(\mathrm{G}, \mathrm{H})$; scale bar $50 \mu \mathrm{m}$ 
Table 1. Least squares means (LSM), standard deviation (SD), and variation (V) for muscle fibre percentage $(\%)$ and diameter $(\varnothing)$, parameters of connective tissue, proportion and content of total and soluble collagen, IMF content and shear force value of $m$. semimembranosus depending on pig breed

\begin{tabular}{l|c|c|c|c|c|c}
\hline \multirow{2}{*}{ Traits } & \multicolumn{3}{c}{ ZS } & \multicolumn{3}{c}{ PL } \\
\cline { 2 - 7 } & LSM & SD & V & LSM & SD & V \\
\hline \%I & $15.8 \mathrm{a}$ & 1.46 & 9.24 & $12.3 \mathrm{~b}$ & 1.06 & 8.62 \\
\%IIA & 13.7 & 2.76 & 20.14 & 12.6 & 2.36 & 18.73 \\
\%IIB & $70.5 \mathrm{a}$ & 2.23 & 3.16 & $75.1 \mathrm{~b}$ & 2.18 & 2.90 \\
ØI $(\mu \mathrm{m})$ & $56.3 \mathrm{a}$ & 1.38 & 2.45 & $59.8 \mathrm{~b}$ & 2.06 & 3.44 \\
ØIIA $(\mu \mathrm{m})$ & 62.4 & 1.97 & 3.16 & 61.9 & 2.54 & 4.10 \\
ØIIB $(\mu \mathrm{m})$ & $73.5 \mathrm{a}$ & 2.63 & 3.58 & $79.9 \mathrm{~b}$ & 2.49 & 3.12 \\
Thickness of endomysium $(\mu \mathrm{m})$ & $4.28 \mathrm{a}$ & 0.32 & 7.48 & $3.49 \mathrm{~b}$ & 0.23 & 6.59 \\
Thickness of perimysium $(\mu \mathrm{mm})$ & $48.94 \mathrm{a}$ & 1.26 & 2.57 & $36.85 \mathrm{~b}$ & 0.92 & 2.50 \\
Collagen area $(\%)$ & $16.65 \mathrm{a}$ & 0.28 & 1.68 & $13.57 \mathrm{~b}$ & 0.24 & 1.77 \\
Collagen content $(\mathrm{mg} / \mathrm{g})$ & $4.98 \mathrm{a}$ & 0.36 & 7.23 & $2.92 \mathrm{~b}$ & 0.26 & 8.90 \\
Soluble collagen $(\%)$ & $18.26 \mathrm{a}$ & 0.83 & 4.54 & $14.26 \mathrm{~b}$ & 1.19 & 8.34 \\
IMF $(\%)$ & $3.28 \mathrm{a}$ & 0.35 & 10.67 & $2.37 \mathrm{~b}$ & 0.36 & 15.19 \\
Shear force $\left(\mathrm{kg} / \mathrm{cm}^{2}\right)$ & $5.83 \mathrm{a}$ & 0.34 & 5.83 & $6.57 \mathrm{~b}$ & 0.42 & 6.39 \\
\hline
\end{tabular}

IMF - intramuscular fat.

$\mathrm{a}, \mathrm{b}-$ least square means (LSM) with different letters differ significantly at $\mathrm{P}<0.05$.

It was found that the $m$. semimembranosus of $\mathrm{ZS}$ pigs was characterized by a significantly greater percentage of type I fibres and a smaller percentage of type IIB fibres compared to the muscles of PL pigs. In addition, ZS pigs had significantly smaller diameters of all the three muscle fibre types compared to PL pigs. In the case of intramuscular connective tissue, the microstructural analysis performed by light and scanning electron microscopy showed every muscle fibre to be surrounded by a thin sheath of wavelike collagen fibres, arranged concentrically around muscle fibres and forming the endomysium. ZS pigs were characterized by a more delicate arrangement of collagen fibres. In the skeletal muscle, muscle fibres are grouped into bundles, which are surrounded by a connective tissue sheath, the perimysium. In the present study, the perimysium of $m$. semimembranosus from ZS pigs is formed by numerous bundles of collagen fibres, which run concentrically and diagonally around muscle bundles and criss-cross to form a relatively loose network. In PL pigs, however, the collagen fibres which form the perimysium are arranged in thick, parallel bundles that adhere tightly together and run concentrically around the muscle bundles. Furthermore, the morphometric analysis showed that both the endomysium and the perimysium were significantly thicker in the $m$. semimembranosus of ZS compared to PL pigs. In turn, the muscles of PL pigs were characterized by a significantly smaller percentage of immunohistochemically detected collagen in the muscle structure, by lower levels of total and soluble collagen, and by lower IMF content compared to the muscles of ZS pigs. As regards shear force, this parameter was significantly lower for the muscles of ZS compared to PL pigs. In the case of variation 
of analysed parameters, percentage of type IIA fibres in m. semimembranosus of ZS and PL pigs was characterized by the highest (about 20\%) variation of all examined parameters. High variation was also observed for IMF content in m. semimembranosus of ZS and PL pigs (over $10 \%$ and $15 \%$, respectively).

\section{Discussion}

Muscle fibre microstructure, composition and size are the most important determinants of pork quality. The greater percentage of type I muscle fibres, found in the present study in the $m$. semimembranosus of ZS pigs, shows that the muscles of these animals are more oxidative than those of PL pigs. These results are typical of primitive pig breeds and meat-type breeds of pigs (Ruusunen and Puolanne, 1997; Serra et al., 1998). These values are also in agreement with the previous results of Bogucka and Kapelański (2005) which reported that ZS pigs compared to PL pigs had a smaller percentage of IIB fibres. Similarly, Bocian et al. (2012) reported that $\mathrm{ZS}$ pigs in $m$. longissimus lumborum had a greater percentage of type I fibres and a lower percentage of type IIB fibres than the muscles of commercial pigs. Lefaucheur et al. (2004) indicate that intensive selection of modern pigs increases the percentage of glycolytic type IIB fibres in muscle fibres compared to the muscles of pigs of local or primitive breeds. Meanwhile, the finer muscle fibres of all three types analysed in the present study in the m. semimembranosus of ZS pigs confirm the earlier observations of Gil et al. (2008), who reported that the muscles of primitive breeds are characterized by smaller fibres. These results are not completely consistent because in the m. longissimus lumborum of Iberico pigs Serra et al. (1998) found type I muscle fibres to be significantly larger compared to the muscles of Landrace pigs. In Lantag and Landrace pigs, Dai et al. (2009) noted no significant differences in muscle fibre size. On the other hand, the smaller muscle fibres in ZS pigs may probably be associated not only with breed but also with the lower body weight of these animals compared to PL pigs. Skeletal muscles are not only muscle fibres. An important element of their structure, which also has a significant effect on the physicochemical parameters of meat, is the structure of intramuscular connective tissue that forms the connective tissue stroma. The stroma is formed by connective tissue sheaths (the endomysium and the perimysium), made up mainly of collagen fibres and elastin surrounded by a proteoglycan matrix. A similar arrangement of collagen fibres in the structure of connective tissue to that observed in the present study in the endomysium and perimysium was described previously by Fang et al. (1999) and Nakamura et al. (2003) in pigs, and by An et al. (2010) in poultry muscles.

High meat quality, which is determined by proper flavour, juiciness and tenderness, depends primarily on IMF content. It is believed that optimum meat flavour is ensured by an IMF content of 2\% (Bejerholm et al., 1986) or 2.5-3\% (DeVol et al., 1988). As is generally known, the meat from pigs of primitive and local breeds, similar to what was observed in the current study for ZS pigs, has a much higher IMF content (Serra et al., 1998; Florowski et al., 2006; Dai et al., 2009) compared to com- 
mercial breeds. This is mainly due to the lower carcass meat content (Daszkiewicz et al., 2005). As reported by Fernandez et al. (1999), IMF level of 3-3.5\%, which is the level found in the present study in ZS pigs, is optimal for making dry-cured products. What is more, the higher IMF content of muscles from ZS pigs may, to a certain extent, be also attributed to muscle fibre composition. Studies by Fiedler et al. (2003) and Wojtysiak and Kaczor (2011) demonstrated that porcine muscles with higher proportions of type I and IIA fibres are characterized by higher levels of IMF. This is related to the fact that the fibres of this type contain much more intracellular fat compared to type IIB fibres.

Tenderness is one of the principal organoleptic attributes that determines the palatability of meat and is an important characteristic in the consumer evaluation of meat quality. Our results for the mechanical measurement of tenderness of $\mathrm{m}$. semimembranosus show the meat of ZS pigs to be more delicate. Muscles that contain large amounts of intramuscular connective tissue, as was the case in ZS compared to PL pigs, are generally tougher, or less tender (Domaradzki et al., 2010). Likewise, An et al. (2010) hold the view that muscles with thicker perimysium are also characterized by higher shear force values. Inverse relationships were found in the present study in which the muscles of ZS pigs, which exhibited a greater proportion of intramuscular connective tissue and thicker endomysium and perimysium, were characterized by lower shear force values compared to the muscles of PL pigs. These differences are probably related to the organization of collagen fibres in the connective tissue stroma. These conjectures are supported by Nishimura et al. (1996), who demonstrated that meat tenderness is determined not only by collagen levels and endomysium and perimysium thickness but also by the size and organization of collagen fibres that form the connective tissue stroma of the muscles. The same authors point out that the muscles in which perimysial structure is made up of thick bundles of collagen fibre that form very compact systems, which we observed in PL pigs, are characterized by higher shear force values. In addition, as shown by Gerrard and Grant (2003), meat tenderness is influenced not so much by total collagen content as by soluble collagen content. This is why the higher level of soluble collagen that we found in ZP pigs may, to a certain extent, explain their lower shear force values despite the greater proportion of both immunohistochemically detected and total collagen. The differences in collagen levels between the analysed breeds of pigs may be also associated with the composition of muscle fibres in the m. semimembranosus, in particular with the higher percentage of type I muscle fibres in ZP pigs. Lepetit (2008) indicates that "white" muscles, which have a greater proportion of type IIB fibres, are characterized by a lower proportion of collagen in muscle structure compared to "red" muscles, the composition of which is dominated by type I muscle fibres. An important role in shaping meat tenderness is also played by intramuscular fat (IMF), which, by being deposited mainly in intramuscular connective tissue, makes it looser, thus affecting the tenderness of meat. The differences in tenderness between the analysed pig breeds may also be attributed to the differences in muscle fibre composition. Cameron et al. (1998) demonstrated positive relationships between shear force values and the percentage of type IIB fibres as well as negative relationships with the percentage of type I fibres, which may explain the greater 
tenderness of meat from Złotnicka Spotted pigs. These findings are not completely consistent because the literature shows inverse relationships. Ryu and Kim (2005) observed the percentage of type I muscle fibres to be positively correlated to shear force. On the other hand, Orzechowska et al. (2008) found no significant correlations between tenderness and composition of muscle fibres. This probably happens because meat tenderness is determined by a number of both pre- and post-slaughter factors. A major role is played by the organization and content of collagen, sarcomere length, water and IMF content, and the content of several proteolytic enzymes, in particular the calpain system enzymes.

In summary, breed of pigs has a significant effect on the structural parameters of m. semimembranosus. The muscles of the local breed of Złotnicka Spotted pigs were more delicate (lower shear force value) and were characterized by finer muscle fibres, more oxidative muscles (greater proportion of type I fibres), greater proportion of total and soluble collagen, higher IMF levels, and a thicker but looser connective tissue stroma compared to the muscles of PL pigs.

\section{References}

An J.Y., Zheng J.X., Li J.Y., Zeng D., Qu L.J., Xu G.Y., Yang N. (2010). Effect of myofiber characteristics and thickness of perimysium and endomysium on meat tenderness of chickens. Poultry Sci., 89: 1750-1754.

AOAC (2000). Official methods of analysis (17th ed.). Gaithersburg, MD: Association of Official Analytical Chemists.

B e jer h o $1 \mathrm{~m} \mathrm{C.,} \mathrm{B} \mathrm{a} \mathrm{r} \mathrm{t} \mathrm{o} \mathrm{n-G} \mathrm{a} \mathrm{d} \mathrm{e} \mathrm{P.A.} \mathrm{(1986).} \mathrm{Effect} \mathrm{of} \mathrm{intramuscular} \mathrm{fat} \mathrm{level} \mathrm{on} \mathrm{eating} \mathrm{quality} \mathrm{of}$ pig meat. Proc. 30th European Meeting of Meat Research Workers, Bristol, pp. 389-391.

B o c i an M., Wojtys iak D., J ankowiak H., C ebulska A., Kapelański W., Migdał W. (2012). Carcass, meat quality and histochemical traits of $m$. longissimus lumborum from Złotnicka Spotted pigs and commercial pigs. Folia Biol. (Krakow), 60: 181-187.

B o g u c k a J., K a p e lańs ki W. (2005). Microstructure of longissimus lumborum muscle in pigs of several breeds and its relation to meat quality traits. Folia Biol. (Kraków), 55, Suppl.: 85-90.

Cam eron N.D., Ok s bjerg N., Hen ckel P., Nute G., B rown S., Wood J.D. (1998). Relationships between muscle fibre traits with meat and eating quality in pigs. Proc. BSAS Annual Meeting, p. 123.

D a i F., F eng D., C a o Q., Ye H., Z hang Ch., X i a W., Z u o J. (2009). Developmental differences in carcass, meat quality and muscle fibre characteristics between the Landrace and Chinese native pig. S. Afr. J. Anim. Sci., 39: 267-273.

D a s z k i ew i c z T., B ą k T., D en aburski J. (2005). Quality of pork with different intramuscular fat (IMF) content. Pol. J. Food Nutr. Sci., 1: 31-36.

DeVol D.L., McKeith F.K., Bechtel P.J., Novakofski J., Shanks R.D., Carr T.R. (1988). Variation in composition and palatability traits and relationships between muscle characteristics and palatability in random sample of pork carcasses. J. Anim. Sci., 66: 385-395.

Domaradzki P., Skałecki P., F lorek M., Litwińczuk Z. (2010). Relationship between collagen and selected technological parameters of veal meat (in Polish). Żywność. Nauka. Technologia. Jakość, 4: 50-62.

Fang S.H., Nishimura T., Takahashi K. (1999). Relationship between development of intramuscular connective tissue and toughness of pork during growth of pigs. J. Anim. Sci., 77: $120-130$.

Fernandez X., Monin G., Talmant A., Mourot J., Lebret B. (1999). Influence of intramuscular fat content on the quality of pig meat. 1. Composition of the lipid fraction and sensory characteristics of $m$. longissimus lumborum. Meat Sci., 53: 59-65. 
Fie d l e r I., N urnberg K., H ard e T., N urnberg G., Ender K. (2003). Phenotypic variations of muscle fibre and intramuscular fat traits in Longissimus muscle of F2 population Duroc $\times$ Berlin Miniature Pig and relationships to meat quality. Meat Sci., 63: 131-139.

Florows ki T., P is u la A., A d a m c za k L., B u c z yń s ki J., Orze c how s ka B. (2006). Technological parameters of meat in pigs of two Polish local breeds - Zlotnicka Spotted and Pulawska. Anim. Sci. Pap. Rep., 24: 217-224.

Gerrard D.E., Grant A.L. (2003). Principles of animal growth and development. Kendall/Hunt Publishing.

Gil M., Delday I., Gispert M., Furnols M., Maltin C.M., Plas tow G.S., Klont R., S o s n i cki A., C a r ri on D. (2008). Relationships between biochemical characteristics and meat quality of Longissimus thoracis and Semimembranosus muscles in five porcine lines. Meat Sci., 80: 927-933.

I w a moto H., Tabata S., Sakakibara K., Nishimura S., Gotoh T., Koga Y. (2001). Scanning electron microscopic observation of the architecture of collagen fibres in chicken M. iliotibialis lateralis. Brit. Poultry Sci., 42: 321-326.

Lefaucheur L., Milan D., Ecolan P., Le Callennec C. (2004). Myosin heavy chain composition of different skeletal muscles in Large White and Meishan pigs. J. Anim. Sci., 82: 1931-1941.

Lepetit J. (2008). Collagen contribution to meat toughness: Theoretical aspects. Meat Sci., 80: $960-967$.

L i u A., N is himura T., Takahash i K. (1996). Relationship between structural properties of intramuscular connective tissue and toughness of various chicken skeletal muscles. Meat Sci., 43: 43-49.

Na ka mura Y.N., I wa mo to H., Ono Y., Sh ib a N., N is himura S., Tabata S. (2003). Relationship among collagen amount, distribution and architecture in the M. longissimus thoracis and M. pectoralis profundus from pigs. Meat Sci., 64: 43-50.

Nish i mura T., Oj i ma K., Li u A., Hattori A., Takahashi K. (1996). Structural changes in the intramuscular connective tissue during development of bovine semitendinosus muscle. Tissue Cell., 28: 527-536.

Orzechowska B., Wojtysiak D., Migdał W., Tyra M. (2008). Relationships between muscle fibre characteristics and physico-chemical properties of longissimus lumborum muscle and growth rate in pig fatteners of three breeds. Anim. Sci. Pap. Rep., 26: 277-285.

PN-ISO 3496/2000. Meat and meat preparations. Determination of hydroxyproline content (in Polish).

Ruusunen M., Puolanne E. (1997). Comparison of histochemical properties of different pig breeds. Meat Sci., 45: 119-125.

R y u Y.C., K i m B.C. (2005). The relationship between muscle fiber characteristics, postmortem metabolic rate, and meat quality of pig longissimus dorsi muscle. Meat Sci., 71: 351-357.

S erra X., Gil F., P erez - En c is o M., Oliver M.A., Vazque z J.M., G is per t M., D i a z I., Moreno F., L at orre R., N oguera J.L. (1998). A comparison of carcass, meat quality and histochemical characteristics of Iberian (Guadyerbas line) and Landrace pigs. Liv. Prod. Sci., 56: $215-223$.

We g n e r J., F i e d l e r I., Kło s ow s k a D., Kło s o w s k i B., Z i e g a n B. (1993). Veränderungen der Muskelfasertypenverteilung im M. longissimus dorsi von Ebern während des Wachstums dargestellt mit verscheidenen histochemischen Methoden. Anat. Histol. Embryol., 22: 355-359.

W o j t y s i a k D., K a c z o r U. (2011). Effect of g.2728G $>$ A and g.3996T $>$ C polymorphisms at the leptin gene locus on microstructure and physicochemical properties of longissimus lumborum muscle of Polish Landrace pigs. Folia Biol. (Krakow), 59: 77-82.

Received: 30 IX 2013

Accepted: 3 III 2014 